\title{
Atendimento multidisciplinar para a educação especial e inclusiva de uma criança com transtorno do espectro autista: um estudo de caso
}

\author{
Multidisciplinary care for the special and inclusive education of a child with autism \\ spectrum disorder: case report
}

\begin{abstract}
Atención multidisciplinaria para la educación especial e inclusiva de un niño con trastorno del espectro autista: informe de caso
\end{abstract}

Mayara Macedo Melo ${ }^{1,2}$, Francisco Lucas de Lima Fontes ${ }^{1 *}$, Ester Lima de Sousa ${ }^{2}$, Elbson Alves e Sousa ${ }^{2}$, Franciane Santos do Nascimento ${ }^{2}$, Geane Blenda Mendes de Andrade ${ }^{2}$, Débora Laís Miranda de Araújo $^{2}$, Paula Alves Sepúlveda Almendra ${ }^{2}$, Elisalma Vieira Carvalho ${ }^{2}$, Stefanny Christina Ribeiro do Nascimento², Maria das Graças Sampaio², Bárbara Bruna dos Santos Silva², Suzana Lima de Sousa ${ }^{2}$, Fernanda Gomes do Nascimento Silva ${ }^{2}$, Ester Samanta Brito de Oliveira ${ }^{2}$.

\section{RESUMO}

Objetivo: Relatar o caso de uma criança com Transtorno do Espectro Autista frente ao atendimento multidisciplinar para a educação especial e inclusiva. Detalhamentos de caso: L. I. S. B., sexo masculino, nascido de parto cesáreo a termo. Durante a gestação a mãe não apresentou intercorrências, porém aos três anos de idade os pais perceberam a dificuldade que a criança possuía na fala e socialização. Após criteriosa avaliação chegou-se ao diagnóstico de Transtorno do Espectro Autista. Em meio ao impacto familiar causado pelo desfecho da investigação, o infante passou a ser acompanhado continuamente por uma equipe multidisciplinar que contava com psicólogo, fonoaudiólogo, neuropediatra e terapeuta ocupacional. A criança ingressou na escola aos três anos de idade, contudo enfrentou inúmeros desafios como a resistência da direção da instituição em recebê-lo, pois o despreparo dos profissionais impedia que o mesmo tivesse acesso ao ensino. Considerações finais: Revela-se a necessidade de maior preparo dos profissionais da educação no convívio com criança autista ao evidenciar desconhecimento e certo preconceito destes no relacionamento vivenciado a princípio. A qualificação de professores e demais profissionais, além de infraestrutura adequada por meio do fornecimento de insumos necessários ao progresso da criança com o transtorno mostram-se essenciais ao ensino-aprendizagem.

Palavras-chave: Transtorno do espectro autista, Educação especial, Estudo de caso.

\begin{abstract}
Objective: To report the case of a child with Autistic Spectrum Disorder facing multidisciplinary care for special and inclusive education. Case report: L. I. S. B., male, born at term cesarean delivery. During pregnancy, the mother did not present any complications, but at three years of age the parents realized the difficulty the child had in speech and socialization. After careful evaluation, we reached the diagnosis of Autism Spectrum Disorder. In the midst of the family impact caused by the outcome of the investigation, the infant was continuously followed up by a multidisciplinary team that counted with a psychologist, speech pathologist, pediatric neurological doctor and occupational therapist. The child entered the school at the age of three but
\end{abstract}

${ }^{1}$ Faculdade UNINASSAU - Campus Redenção. Teresina, Piauí. *E-mail: lucasfontesenf@hotmail.com
2 Universidade Federal do Piauí. Teresina. Brasil.

SUBMETIDO EM: $3 / 2019$

ACEITO EM: 4/2019

PUBLICADO EM: 6/2019

REAS/EJCH | Vol. Sup. 25 | e589 | DOI: https://doi.org/10.25248/reas.e589.2019 Página 1 de 6 
faced numerous challenges such as the resistance of the board of directors to receive it, because the unprepared professionals prevented it from having access to education. Final considerations: It is revealed the need for greater preparation of the professionals of the education in the conviviality with autistic child, as evidencing their ignorance and certain prejudice in the relationship experienced at the beginning. The qualification of teachers and other professionals, as well as adequate infrastructure through the supply of necessary inputs to the progress of the child with the disorder are essential to teaching learning.

Keywords: Autism spectrum disorder, Special education, Case report.

\section{RESUMEN}

Objetivo: Informar el caso de un niño con trastorno del espectro autista frente a la atención multidisciplinaria para la educación especial e inclusiva. Informe de caso: L. I. S. B., sexo masculino, nacido de parto cesáreo a término. Durante la gestación la madre no presentó intercurrencias, pero a los tres años los padres percibieron la dificultad que el niño poseía en el habla y socialización. Después de una cuidadosa evaluación se llegó al diagnóstico de Trastorno del Espectro Autista. En medio del impacto familiar causado por el desenlace de la investigación, el infante pasó a ser acompañado continuamente por un equipo multidisciplinario que contaba con psicólogo, fonoaudiólogo, neuropediatra y terapeuta ocupacional. El niño ingresó en la escuela a los tres años de edad, sin embargo enfrentó innumerables desafíos como la resistencia de la dirección de la institución a recibirlo, pues la despreparación de los profesionales impedía que el mismo tuviera acceso a la enseñanza. Consideraciones finales: Se revela la necesidad de una mayor preparación de los profesionales de la educación en la convivencia con niño autista al evidenciar desconocimiento y cierto prejuicio de éstos en la relación vivenciada al principio. La cualificación de profesores y demás profesionales, además de infraestructura adecuada por medio del suministro de insumos necesarios para el progreso del niño con el trastorno, son esenciales para la enseñanza-aprendizaje.

Palabras-clave: Trastorno del espectro autista, Educación especial, Informe de caso.

\section{INTRODUÇÃO}

O Transtorno do Espectro Autista (TEA) engloba modificações no padrão de desenvolvimento neurológico. É perceptível normalmente a partir do terceiro ano de vida e afeta a capacidade de socialização e de comunicabilidade, predispondo o sujeito a comportamentos estereotipados. Abrange aqueles que apresentem desde deficiência intelectual grave até indivíduos com quociente de inteligência padrão (OLIVEIRA KG, SETIÉ AL, 2017).

A Organização Mundial da Saúde (OMS) define o TEA como uma disfunção do desenvolvimento, incurável e rigorosamente incapacitante, com incidência de cinco casos a cada 100.000 nascidos vivos (BRITO ER, 2015).

As dificuldades apresentadas pelo sujeito autista comprometem o processo de ensino-aprendizagem. Contudo tais obstáculos não impedem a educação e inclusão da criança na escola regular. Para isso, devem Ihe ser oferecidas condições necessárias como equipe técnica preparada e qualificada na utilização de métodos e programas que utilizem metodologias de ensino específicas, recursos visuais adaptativos, atividades recreativas motoras e organização da área física. Ademais, o programa Currículo Funcional Natural possibilita aprimorar aptidões que tornam o sujeito autista autônomo e criativo, favorecendo sua aprendizagem (GIARDINETTO ARSB et al, 2013).

Dentro das políticas públicas que embasam a educação especial ressaltam-se a Lei no 9.394 de Diretrizes e Bases da Educação de 1996, a Declaração de Salamanca de 1994 e a Declaração Universal dos Direitos Humanos de 1948. Tais políticas salientam a igualdade e o direito que o indivíduo possui à educação na escola regular de ensino, o que inclui a criança com TEA (SERRA D, 2010). 
A criança quando entra na creche tem a oportunidade de desenvolver seu intelecto e consequentemente as relações sociais. Para a criança diagnosticada com TEA, esse primeiro contato é pontuado como um momento de grande importância para a sua inclusão e principalmente desenvolvimento, uma vez que a escola deve oferecer um ambiente simplório, sem grandes complexidades assim como adaptação curricular da instituição, ancorada no apoio e comprometimento familiar (BRITO ER, 2015).

Diante desse contexto, o objetivo deste estudo foi relatar o caso de uma criança com Transtorno do Espectro Autista frente ao atendimento multidisciplinar para a educação especial e inclusiva.

\section{DETALHAMENTO DO CASO}

O objeto de estudo da presente pesquisa trata-se de L. I. S. B., sexo masculino, nascido de parto cesáreo a termo. Durante a gestação a mãe não apresentou intercorrências, porém aos três anos de idade os pais perceberam a dificuldade que a criança possuía na fala e socialização. A coleta das informações ocorreu no município de Teresina, capital do Piauí, no período de março a junho de 2018 por meio de observação e questionamentos abertos aos pais da criança.

No primeiro atendimento com o médico pediatra, após avaliação, a criança foi encaminhada ao fonoaudiólogo para solução do problema de afasia. Nesse contato, o especialista identificou a viabilidade do desenvolvimento de fala da criança. Pela desconfiança de que ele poderia possuir o TEA, o profissional o encaminhou para consulta com neuropediatra. Após criteriosa avaliação chegou-se ao diagnóstico de TEA. Em meio ao impacto familiar causado pelo desfecho da investigação, L. I. S. B. passou a ser acompanhado continuamente por uma equipe multidisciplinar que contava com psicólogo, fonoaudiólogo, neuropediatra e terapeuta ocupacional.

Após consultas e auxílios dos profissionais, L. I. S. B. conseguiu pronunciar suas primeiras palavras. Com essa leve, porém significativa evolução, os pais do garoto procuraram alternativas de acompanhamento no Centro Integrado de Reabilitação, Centro Integrado de Educação Especial e na Associação de Pais e Amigos de Deficientes Auditivos (APADA), obtendo maior êxito nesta última.

Além da APADA, L. I. S. B. também era acompanhado duas vezes por semana com uma psicopedagoga de atendimento educacional especializado. Neste atendimento, trabalhava-se sobretudo a coordenação motora, ocasião em que foi implementada uma tecnologia assistiva com a adaptação do lápis para que ele pudesse escrever e melhor se desenvolver.

Até o momento de coleta das informações, L. I. S. B. fazia uso de risperidona diariamente, medicação da qual era dependente. Até encontrar o medicamento correto, a criança fez uso de carbamazepina, porém os efeitos colaterais deste fármaco trouxeram maléficos como agitação, agressividade e insônia.

L. I. S. B. ingressou na escola aos três anos de idade, mesmo período em que foi diagnosticado com TEA. Nesta época o infante ainda fazia uso da carbamazepina, fármaco que não o beneficiava, e em seus primeiros dias na escola o aluno não conseguia concentrar-se nas aulas, atrapalhando os demais, além de apresentação de descontrole emocional constante. Diante de tais circunstâncias a criança acabou sendo afastada da escola até adaptar-se ao novo medicamento.

Após a estabilização de sua condição, retornou à instituição e enfrentou novos desafios como a resistência da direção em recebê-lo, pois o despreparo dos profissionais o impedia de ter acesso ao ensino. Posterior a discussões, L. I. S. B. conseguiu seu direito de frequentar a escola. Apesar de todas as dificuldades enfrentadas, o aluno desenvolveu-se bem nos exercícios propostos, participando de atividades culturais e evidenciando capacidades de desenvolvimento.

Tempos depois, por motivos logísticos houve a necessidade de troca de escola. A criança passou por momentos de intenso estresse, pois para pessoas desse grupo de transtorno a rotina torna-se um fator protetivo, de modo que facilita seu desenvolvimento. Nessa transição, L. I. S. B sofreu preconceito e foi excluído do convívio pelos demais alunos, professores e funcionários da nova escola, prejudicando assim seu quadro clínico, o que afetou seu aproveitamento educacional. Por um longo período, o colégio não ofereceu 
a assistência necessária para o desenvolvimento do aluno, resultando em prejuízo cognitivo e educativo, pois não havia auxiliar de inclusão para acompanhá-lo e norteá-lo durante as atividades acadêmicas.

Mediante a situação conflituosa e desfavorável ao qual L. I. S. B. estava submetido, sua mãe procurou meios legais para que a escola o acolhesse com as devidas condições que ele necessitava. A direção da instituição, pressionada a prestar um serviço de qualidade ao aluno autista, recorreu a Secretaria Municipal de Educação ao qual se aloca e solicitou uma acompanhante especializada para auxiliá-lo em suas atividades.

A pedagoga que acompanhava L. I. S. B. estava há sete meses conduzindo as medidas metodológicas e técnicas realizadas com o infante. Após a sua entrada na escola, implementou os encontros de atendimento educacional especializado e produziu exercícios e atividades que condiziam com o mesmo conteúdo ministrado para as demais crianças, adaptando e explorando as habilidades e preferências do aluno como a utilização de cores e animais preferidos.

A pedagoga procurou qualificar-se e compreender melhor o transtorno sofrido pelo aluno e a educação especial na prática, aperfeiçoando assim suas práticas pedagógicas inclusivas. $O$ trabalho conjunto da pedagoga com a professora resultou em evolução de L. I. S. B. nos aspectos motor, intelectual e afetivo. As TA utilizadas facilitavam o trabalho da pedagoga da escola com L. I. S. B por proporcionar alternativas práticas que ampliavam a utilização de materiais essenciais no processo de ensino-aprendizagem.

Até o período de coleta das informações, L. I. S. B. conseguia acompanhar o ritmo de conteúdos da turma, resultado principalmente das adequações feitas pela pedagoga. Todavia, o aluno enfrentava o obstáculo da coordenação motora fina ainda não totalmente desenvolvida, que impedia a pega correta de objetos de menor espessura, como o lápis, principalmente.

\section{DISCUSSÃO}

O diagnóstico interdisciplinar é encarado como uma das maneiras mais seguras de se identificar e tratar - TEA, com a inserção de ao menos psicólogo e neuropediatra especializados em distúrbios do desenvolvimento. A atuação desses profissionais permite a visualização de nuances do quadro clínico apresentado pela criança com o transtorno e possibilita à família informações específicas sobre cada campo de atuação dos profissionais envolvidos na implementação do tratamento e acompanhamento da criança autista (SILVA M e MULICK JA, 2009).

A primeira intervenção terapêutica no caso do TEA é a abordagem psicossocial e intervenções educacionais, com a finalidade de ampliar a aquisição e o aumento do repertório de linguagem, assim como melhorar as habilidades sociais e findar comportamentos mal adaptativos. Se tratando da terapia farmacológica, não existe um medicamento padrão disponível que repercuta sobre as causas do transtorno, assim utilizando medicamentos conforme a singularidade do caso (NIKOLOV R et al., 2006).

No caso de L. I. S. B. dois fármacos foram utilizados. A risperidona pertence à classe dos antipsicóticos atípicos. Este medicamento vem sendo prescrito por neuropediatras com o intuito de diminuir comportamentos auto lesivos, agressividade, estereotipias e crises de ira. A carbamazepina pertence à classe dos anticonvulsivantes, normalmente aplicada em pessoas com autismo devido alta incidência de convulsões neste grupo, mas também para minimizar os episódios de agressividade e descontrole comportamental (OLIVEIRA FCA et al., 2015).

De modo a garantir pleno desenvolvimento cognitivo e social, além do tratamento medicamento necessário ao controle emocional e concentração da criança, pode-se empregar também o recurso de tecnologias assistivas, que funcionam como um auxílio ao progresso do indivíduo autista ao propiciar uma melhor qualidade de vida por intermédio de inclusão do sujeito nos meios sociais (CARNEIRO VB et al., 2015).

A tecnologia assistiva é pertinente no contexto educacional, ao tornar-se imprescindível para a escola em atividades desenvolvidas com alunos da educação especial. Nela, são utilizados todo o arsenal de recursos e instrumentos disponíveis para que seja suprida as necessidades e deficiências de seus alunos permitindoIhes ter maior autonomia na realização de atividades (SANTANA LS et al., 2012). 
As crianças com TEA necessitam de acompanhamento contínuo, contudo as instituições prestadoras dessa assistência enfrentam desafios como a falta de recursos financeiros, necessários ao atendimento efetivo e qualificação profissional. Atualmente, no Brasil, as iniciativas governamentais de apoio ao indivíduo diagnosticado com TEA acontecem de modo tardio. No início dos anos 2000, este público encontrava atendimento especializado apenas em instituições filantrópicas como a Associação dos Pais e Amigos dos Excepcionais (APAE) ou em instituições não governamentais idealizadas, desenvolvidas e mantidas por familiares de sujeitos autistas (OLIVEIRA DBC et al., 2017).

A ausência de recursos e entidades públicas voltadas ao apoio de autistas e suas famílias obrigou que pais tomassem iniciativas próprias, como a formação de círculos de divulgação de conhecimentos e auxílio mútuo, espelhados em experiências de outros países. Somente anos depois, a movimentação de familiares levou a aprovação de uma lei federal específica que amparava o sujeito com TEA (MELLO AM et al., 2013).

A Lei № 12.764, de 27 de dezembro de 2012, institui a Política Nacional de Proteção dos Direitos da Pessoa com Transtorno do Espectro Autista e incide em âmbitos assistencial, político, científico e educacional/pedagógico. Como forma de garantir acesso ao ensino a pessoas com TEA, seu artigo $7^{\circ}$ decreta que:

"O gestor escolar, ou autoridade competente, que recusar a matrícula de aluno com transtorno do espectro autista, ou qualquer outro tipo de deficiência, será punido com multa de 3 (três) a 20 (vinte) salários-mínimos" (BRASIL, 2012).

A Lei caracterizou um marco da busca de direitos do sujeito autista, de forma que a incorporação da doença na esfera das deficiências possibilita acesso a direitos previstos em lei já existentes a indivíduos com deficiência no país, além facilitar acesso aos serviços de atendimento especializados (NUNES F e ORTEGA F, 2016).

Referente à educação especial dessa criança, é necessária intervenção segura e satisfatória em parceria com os atores profissionais atuantes (professor, acompanhante especializado e pedagogo). $O$ compartilhamento de experiências, a qualificação na educação especial e inclusiva e o esforço na pesquisa de técnicas de manejo do aluno são estratégias que podem ser adotadas pelos profissionais (PEREIRA CAV et al., 2013).

O pedagogo planeja as atividades desenvolvidas com o aluno autista a partir de conhecimentos prévios discutidos com outros profissionais acerca do perfil, preferências e gostos mais acentuados do aluno de modo que potencialize a absorção de conhecimentos e conteúdos propostos (SCHMIDT C et al., 2016).

A rotina do autista deve ser levada em consideração, pois a partir dela ele consegue desenvolver uma organização têmporo-espacial do ambiente, o que facilita sua aprendizagem (GIARDINETTO ARSB et al, 2013).

O respeito as especificidades do aluno autista bem como a estimulação dessa criança, por meio de suas habilidades e preferências, refletem diretamente no seu desenvolvimento e na evolução da sua aprendizagem, mas especialmente sobre os aspectos cognitivos, emocionais e sociais (BUSATO SCC, 2016).

\section{CONSIDERAÇÕES FINAIS}

Embora não haja pela medicina uma etiologia definida para o Transtorno do Espectro Autista, atualmente conta-se com inúmeras terapias alternativas voltadas ao controle e melhoria da qualidade de vida da criança acometida por esse transtorno. Percebe-se também avanços legislativos e tecnológicos que incluem a criança com a patologia na sociedade e principalmente no ambiente escolar, propiciando dessa forma seu pleno desenvolvimento cognitivo e social. $O$ estudo também revela a necessidade de maior preparo dos profissionais da educação no convívio com criança autista ao evidenciar desconhecimento e certo preconceito destes no relacionamento vivenciado a princípio. A qualificação de professores e demais profissionais, além de infraestrutura adequada por meio do fornecimento de insumos necessários ao progresso da criança com o transtorno mostram-se essenciais ao ensino-aprendizagem. 


\section{REFERÊNCIAS}

1. BRASIL. Lei $n^{\circ}$ 12.764, de 27 de dezembro de 2012. Institui a Política Nacional de Proteção dos Direitos da Pessoa com Transtorno do Espectro Autista; e altera o $\S 30$ do art. 98 da Lei no 8.112, de 11 de dezembro de 1990, Brasília, 2012.

2. BRITO ER. A inclusão do autista a partir da educação infantil. Revista Eventos Pedagógicos, 2015; 6(2): 82-91.

3. BUSATO SCC. Estratégias facilitadoras para o ensino de matemática no ensino fundamental para crianças do espectro autista. Revista Científica Intelletto, 2016; 1(3): 163-171.

4. CARNEIRO VB et al. A tecnologia assistiva no processo de mediação da aprendizagem do aluno autista. In: XII Congresso Nacional de Educação (EDUCERE). Pontifícia Universidade Católica do Paraná, 2015: $7391-7407$.

5. GIARDINETTO ARSB et al. O professor da Educação Especial e o processo de ensino-aprendizagem de alunos com autismo. Revista Educação Especial, 2013; 26(46): 385-400.

6. MELLO AM et al. Retratos do autismo no Brasil. 1a ed. São Paulo: Associação dos Amigos do Autista, 2013.

7. NIKOLOV R et al. Autismo: tratamentos psicofarmacológicos e áreas de interesse para desenvolvimentos futuros. Revista Brasileira de Psiquiatria, 2006; 28(1): 39-46.

8. NUNES F, ORTEGA F. Ativismo político de pais de autistas no Rio de Janeiro: reflexões sobre o "direito ao tratamento". Saúde e Sociedade, 2016; 25(4): 964-975.

9. OLIVEIRA DBC et al. Políticas para o autismo no Brasil: entre a ação psicossocial e a reabilitação. Revista de Saúde Coletiva, 2017; 27(3): 707-726.

10. OLIVEIRA FCA et al. Perfil farmacêutica de crianças autistas de uma clínica de reabilitação no estado do Ceará. Boletim Informativo Geum, 2015; 6(3): 43-49.

11. OLIVEIRA KG, SERTIÉ AL. Transtorno do espectro autista: um guia atualizado para aconselhamento genético. Einstein, 2017; 15(2): 233-238.

12. PEREIRA CAV et al. Autismo infantil: aplicações do ensino estruturado na inclusão escolar. Revista de Ciências da Saúde Nova Esperança, 2013; 11(3): 75-82.

13. SANTANA LS. Inclusão escolar: a utilização da tecnologia assistiva na educação regular. In: II Simpósio Educação e Comunicação. Universidade Federal de Sergipe, 2012: 345-355.

14. SCHMIDT C et al. Inclusão escolar e autismo: uma análise da percepção docente e práticas pedagógicas. Revista Psicologia: Teoria e Prática, 2016; 18(1): 222-235.

15. SERRA D. Sobre a inclusão de alunos com autismo na escola regular. Quando o campo é quem escolhe a teoria. Revista de Psicologia, 2010; 1(2): 163-176.

16. SILVA M, MULICK JA. Diagnosticando o transtorno autista: aspectos fundamentais e considerações práticas. Psicologia Ciência e Profissão, 2009; 1(29): 116-131. 